\title{
Characterization of human sperm populations using conventional parameters, surface ubiquitination, and apoptotic markers
}

\author{
Sandra Varum, B.Sc., ${ }^{\mathrm{a}}$ Carla Bento, B.Sc., ${ }^{\mathrm{a}}$ Ana Paula M. Sousa, B.Sc., ${ }^{\text {a }}$ Carina S. S. Gomes- \\ Santos, B.Sc., ${ }^{\text {a }}$ Paula Henriques, B.Sc., ${ }^{\mathrm{b}}$ Teresa Almeida-Santos, Ph.D., M.D., ${ }^{\mathrm{b}}$ \\ Cristina Teodósio, B.Sc., ${ }^{\mathrm{c}}$ Artur Paiva, Ph.D. ${ }^{\mathrm{c}}$ and João Ramalho-Santos, Ph.D. ${ }^{\mathrm{a}}$ \\ ${ }^{a}$ Center for Neuroscience and Cell Biology, Department of Zoology, University of Coimbra, Coimbra, Portugal; ${ }^{\mathrm{b}}$ Department \\ of Maternal-Fetal Medicine, Genetics and Human Reproduction, University Hospitals of Coimbra, Coimbra, Portugal; and \\ ${ }^{\mathrm{c}}$ Histocompatibility Centre of Coimbra, Coimbra, Portugal
}

\begin{abstract}
Objective: To directly compare distinct assays proposed to monitor human sperm quality and possibly preselect sperm populations for assisted reproductive technology (ART).

Design: Analysis of human sperm sample quality using several methodologies.

Setting: Academic and clinical institutions.

Patient(s): Samples from consenting patients undergoing routine semen analysis or ART.

Interventions: Human sperm samples were analyzed in terms of World Health Organization parameters and processed for annexin $\mathrm{V}$, terminal deoxynucleotidyl transferase-mediated dUTP nick-end labeling of DNA (TUNEL), and the sperm-ubiquitin tag immunoassay (SUTI). Samples were analyzed both by flow cytometry and fluorescence microscopy.

Main Outcome Measure(s): Correlations among apoptotic markers (outer leaflet phosphatidylserine exposure, membrane integrity, and DNA fragmentation), external ubiquitination, and semen parameters in human spermatozoa.

Result(s): Nonviable sperm, TUNEL-positive cells, and ubiquitin fluorescence intensity means inversely correlate with semen parameters. Apoptotic markers do not correlate with sperm surface ubiquitination. Normozoospermic samples have a higher number of viable cells and lower DNA fragmentation compared with samples with abnormal parameters. Nonviable sperm are more prevalent in samples with low counts and poor morphology but not low motility. Not all sperm with morphologic abnormalities present surface ubiquitination.

Conclusion(s): Sperm quality is inversely correlated with lack of viability, DNA fragmentation, and ubiquitin fluorescence intensity means. However, none of the apoptotic markers correlate with ubiquitin labeling. Elimination of defective sperm cells prior to ART using surface markers (annexin V, ubiquitin) seems unwarranted at this stage. (Fertil Steril ${ }^{\circledR}$ 2007;87:572-83. @2007 by American Society for Reproductive Medicine.)
\end{abstract}

Key Words: human sperm, semen parameters, annexin V, TUNEL, ubiquitin, fluorescence microscopy, flow cytometry

The heterogeneous nature of every ejaculate in terms of sperm functionality has led to many attempts both to characterize distinct populations and to improve sorting/ selection of male gametes for assisted reproductive technology (ART).

Besides classical techniques (gradient centrifugation, swim-up), more recent assays have been proposed based on differential staining of spermatozoa with biologically relevant markers. In this case, following classical separations, the cells would then be further incubated with beads conjugated with different probes that would bind defective sper-

Received October 5, 2005; revised and accepted 21 July 2006.

Supported by a grant from the Portuguese National Science Foundation (Fundação para a Ciência e Tecnologia, FCT) to J.R.-S. (POCTI/ESP/ 38049/2001). S.V. and A.P.S. are supported by Ph.D. grants from FCT, Portugal.

Reprint requests: João Ramalho-Santos, Ph.D., Center for Neuroscience and Cell Biology, Department of Zoology, University of Coimbra, 3004-517 Coimbra, Portugal (FAX: +351 239 855789; E-mail: jramalho@ci.uc.pt). matozoa not yet eliminated. Removal of the beads (together with bound cells) would thus yield a theoretically higher quality sample. This would be especially relevant if the defective sperm cells in question could not be identified by simple visualization (1). Indeed, although spermatozoa from patients possess multiple structural and functional defects, usually evaluated by light microscopy (2), this approach can be limited, because, for example, spermatozoa with normal morphology can, nevertheless, have DNA defects or be positive for apoptotic markers (3).

Apoptotic markers seem to be good candidates for the possible selection of functional sperm (or for the removal of abnormal cells). Apoptosis is a process of cell death based on genetic mechanisms that induce a series of cellular, morphologic, and biochemical alterations (4). It is, for example, involved in counteracting clonal expansion during spermatogenesis $(5,6)$. Furthermore, male fertility can be affected by alterations in apoptotic mechanisms (7). 
One of the earliest apoptotic events is the translocation of the phospholipid phosphatidylserine (PS) from the inner to the outer leaflet of the plasma membrane. PS exposure provides an opportunity to identify cells that are in an early stage of apoptosis. These cells can be detected using fluorescently tagged annexin $\mathrm{V}$, which binds PS in a calciumdependent fashion, and has thus been used to detect apoptotic spermatozoa (7-9). When coupled with an impermeant DNA dye, the annexin $\mathrm{V}$ assay can also, in parallel, quantify membrane integrity.

Another important feature of apoptosis is DNA fragmentation. This process occurs in the later stages of apoptosis, results from the activation of endogenous endonucleases (9), and can be detected using a variety of assays, such as the sperm chromatin structure assay (SCSA), comet, or the terminal deoxynucleotidyl transferase-mediated dUTP nickend labeling assay, known as the TUNEL assay (3). Several authors have proposed DNA strand breaks as the main evidence for apoptosis in human spermatozoa $(4,8,10)$. However, it is important to note that DNA fragmentation in mature sperm can have other origins besides apoptosis, as it can occur during or after DNA packaging in spermiogenesis or as a result of oxidative stress (8).

Although several reports refer to DNA fragmentation and externalization of PS in human sperm as putative markers for sperm selection, correlations with seminal parameters (and fertility) are still controversial.

Recently, ubiquitin labeling was validated as a biomarker for human infertility (11). Ubiquitin is a ubiquitously expressed 76-amino-acid protein that can be covalently attached to target proteins, and polyubiquitination tags proteins for degradation by $26 \mathrm{~S}$ proteasome or by lysosomes (12). It was originally proposed (13) that epididymal epithelial cells secrete ubiquitin and phagocyte defective sperm labeled with this marker. This study also demonstrated that ubiquitinated spermatozoa found in the ejaculate, and which may have escaped phagocytosis, are characterized by major defects in the head or axoneme. Furthermore, more recent studies proposed that this biomarker could be used [a] to assess sperm quality by flow cytometry, with poor quality samples yielding higher ubiquitination, which would translate to higher fluorescence intensities (11), and [b] that the assay could also help select spermatozoa for ART, as a complement for classical separations (1).

Surprisingly, results obtained by other authors suggest that sperm ubiquitination positively correlates with normal semen parameters in human sperm (especially morphology), thus implying that there may be a functional role for ubiquitination in normal sperm (14). The same authors have also demonstrated the presence of bodies of different sizes in sperm samples, especially in oligoasthenoteratozoospermic (OAT) men, which can be detected when stained with the Merocyanine 540 (M540) dye. The nature of these bodies is not well defined, but they do not contain DNA and may result from sperm degradation (15). It was thus suggested that the negative correlation found between seminal parameters and ubiquitination by flow cytometry (11) was mainly driven by these bodies and not by sperm (14).

The corollary of these contradictory results is that, although the original proposal to use ubiquitination as a biomarker for male infertility may still be valid, direct applications for sperm selection in the clinic would be unwarranted. Furthermore, the actual biological significance (positive or negative) of human sperm ubiquitination remains elusive.

Our aims in this study were [a] to determine percentages of translocation of PS and DNA fragmentation and correlate them with seminal parameters; [b] to determine percentages of ubiquitination in the same semen samples and correlate them with seminal parameters and both apoptosis assays. To our knowledge, this is the first time these three different assays have been carried out simultaneously on the same samples in humans, although similar work has been performed by our group in feline sperm (16). Given the differences in methodology reported by other authors, we analyzed samples by both direct visualization (fluorescence microscopy) and flow cytometry, in this case quantifying both the percentage of labeled entities and the fluorescence intensity of biomarkers.

\section{MATERIALS AND METHODS}

All chemicals were from Sigma Chemical Company (St. Louis, MO), unless stated otherwise. All patients signed informed consent forms, and all human material was used in accordance with the appropriate ethical and internal review board (IRB) guidelines provided by the University Hospitals of Coimbra.

\section{Biological Material}

Human sperm samples were obtained from the Fertility Clinic (University Hospitals of Coimbra) from patients undergoing routine semen analysis or fertility treatments involving both in vitro fertilization (IVF) and intracytoplasmic sperm injection (ICSI). During a 2-year period, a total of 150 samples were collected and used in this study (see Table 1 for details). Fresh semen samples were obtained by masturbation after 2-5 days abstinence and were allowed to liquefy for 10-20 minutes at room temperature or for 20-30 minutes at $37^{\circ} \mathrm{C}$ in cases of high viscosity. The routine seminal parameters were evaluated according to World Health Organization (WHO) criteria (2).

The variables taken into consideration were total sperm number, volume, $\mathrm{pH}$, leukocyte number, motility, and sperm concentration. For morphologic analysis, semen smears were made on microscopy slides and then treated with the DiffQuick stain set (Dade Behring Inc., Newark, NJ). Sperm morphology was assessed using strict criteria (17). Information on proven fertility of patients was not available. All the 


\begin{tabular}{|c|c|c|c|c|c|}
\hline \multicolumn{6}{|c|}{$\begin{array}{l}\text { Total amount of samples analyzed per group } \\
\text { and assay }\end{array}$} \\
\hline \multirow[b]{2}{*}{ Assay } & \multicolumn{4}{|c|}{ Groups } & \multirow[b]{2}{*}{ Total } \\
\hline & $\mathbf{N}$ & A & $\mathbf{P 2}$ & OAT & \\
\hline Annexin V & 22 & 16 & 19 & 9 & 66 \\
\hline TUNEL & 24 & 16 & 13 & 9 & 62 \\
\hline SUTI & 39 & 25 & 33 & 16 & 113 \\
\hline SUTI + M540 & 14 & 8 & 11 & 4 & 37 \\
\hline $\begin{array}{l}\text { Total number of } \\
\text { samples }^{\text {a }}\end{array}$ & & & & & 150 \\
\hline \multicolumn{6}{|c|}{$\begin{array}{l}\text { a The total number of samples include } 113 \text { samples } \\
\text { used for annexin V, TUNEL, and/or SUTI assays } \\
\text { (most of the samples were used in all three assays, } \\
\text { however this was not always possible) plus } 37 \text { sam- } \\
\text { ples used only in SUTI + M540. }\end{array}$} \\
\hline \multicolumn{6}{|c|}{ Varum. Assays to monitor human sperm quality. Fertil Steril 2007.} \\
\hline
\end{tabular}

fluorescent tests described below were carried out blind by at least two observers.

\section{Flow Cytometry Analysis}

Sperm population was defined after passing samples through a sorter and separating the different populations obtained in a scatterplot of complexity versus size (Fig. 1A). These populations were analyzed and classified as sperm population, small cells/cellular debris population, and large cell population.

\section{Detection of Membrane PS Exposure and Membrane Integrity Using the Annexin V Assay}

The detection of PS externalization was performed using annexin V conjugates (Molecular Probes, Eugene, OR). Semen samples containing $5 \times 10^{6}$ spermatozoa/mL were first washed and centrifuged $(800 \times g$ for 5 minutes $)$ with HEPES buffer (10 mM HEPES, $140 \mathrm{mM} \mathrm{NaCl}, 2.5 \mathrm{mM}$ $\mathrm{CaCl}_{2}, \mathrm{pH}=7.4$ ). After resuspension in HEPES buffer, 5 $\mu \mathrm{L}$ of allophycocyanin-annexin $\mathrm{V}$ and $0.1 \mu \mathrm{L}$ from an 0.005 $\mathrm{mM}$ stock solution of the impermeant DNA dye Green Nucleic Acid Stain (Sytox; Molecular Probes) were added for each $100 \mu \mathrm{L}$ of cellular suspension. Sperm was incubated in this solution for 15 minutes at room temperature, washed again, and centrifuged $(800 \times g$ for 5 minutes). Both positive (induction of apoptosis with $\mathrm{H}_{2} \mathrm{O}_{2}$ ) and negative (HEPES buffer without $\mathrm{Ca}^{2+}$ ) controls were performed. Finally, $500 \mu \mathrm{L}$ of HEPES buffer was added, and the samples were immediately analyzed by flow cytometry using a FACSCalibur flow cytometer (Becton Dickinson, Franklin Lakes, NJ). The settings included a 488- and 635-nm excitation with a 530/30-nm bandpass filter for fluorescein isothiocyanate conjugate (FITC) detection (Sytox) and a 661/ 16-nm bandpass filter for allophycocyanin (annexin V) detection.

At least 10,000 cells in each sample were collected, and the percentages of the following were calculated from the histogram using the Paint-A-Gate and CELL Quest software (Becton Dickinson): intact sperm without any staining (no fluorescence, $\mathrm{An}-; \mathrm{S}-$ ) = viable sperm; intact sperm with externalized PS that stained only with allophycocyaninannexin $\mathrm{V}(\mathrm{red}, \mathrm{An}+; \mathrm{S}-)$ = early apoptosis; damaged sperm with externalized PS that stained both with allophycocyanin-annexin $\mathrm{V}$ and Sytox (red and green, $\mathrm{An}+; \mathrm{S}+$ ) $=$ late apoptosis; and damaged sperm without externalized PS that stained only with Sytox (green, $\mathrm{An}-; \mathrm{S}+$ ) $=$ nonviable sperm. Results of the annexin $\mathrm{V}$ assay on our samples were also analyzed by fluorescence microscopy, in this case using phycoerythrin-annexin V (Molecular Probes) and an Axioplan 2 Imaging fluorescence microscope (Carl Zeiss, Göttingen, Germany) equipped with a triple bandpass filter. The assay was coupled with both Sytox and the membranepermeable DNA dye Hoechst 33342 (Molecular Probes), which allows the detection of viable cells. At least 200 sperm cells were assessed per slide in four fields by at least two different observers. The interobserver and intraobserver variability was $<5 \%$.

\section{DNA Fragmentation Monitored Using the TUNEL Assay}

To monitor DNA fragmentation via the TUNEL assay, we used the APO-BrdU TUNEL assay Kit (Molecular Probes), and the procedure was in accordance with the manufacturer's instructions. Briefly, after sperm fixation with $2 \%$ formaldehyde in phosphate-buffered saline (PBS; $\mathrm{pH}=7.2$ ) for 60 minutes, and permeabilization in PBS with $1 \%$ Triton for 30 minutes, the samples were washed with the kit washing buffer. They were then incubated into a DNA labelingsolution (10 $\mu \mathrm{L}$ of reaction buffer, $0.75 \mu \mathrm{L}$ of TdT enzyme, $8.0 \mu \mathrm{L}$ of BrdUTP, and $31.25 \mu \mathrm{L}$ of $\mathrm{H}_{2} \mathrm{O}$ ) for 60 minutes at $37^{\circ} \mathrm{C}$. The cell suspension was then centrifuged and washed in $300 \mu \mathrm{L}$ of the rinse buffer. After washing, the cell pellets were resuspended in $100 \mu \mathrm{L}$ of the antibody solution (1:20 of Alexa Fluor 488 anti-BrdU antibody diluted in rinse buffer) and incubated for 40 minutes in the dark at room temperature. Both positive (induction of apoptosis with $\mathrm{H}_{2} \mathrm{O}_{2}$ ) and negative (just secondary antibody-488 anti-BrdUTP) controls were performed. The stained cells were analyzed by flow cytometry and fluorescence microscopy on the day of processing.

Percentages of TUNEL-positive cells were obtained using the FACSCalibur cytometer at a $488-\mathrm{nm}$ wavelength. At least 10,000 cells were analyzed per sample, as described above. In order to analyze the samples by fluorescence microscopy, the stained cells were counterstained with 4,6diamino-2-phenylindole (DAPI) (Molecular Probes) before analysis, placed on coverslips, which were mounted in VectaShield mounting medium (Vector Labs, Burlingame, $\mathrm{CA})$, and sealed with nail polish. 


\section{FIGURE 1}

Sperm populations analyzed by flow cytometry using the annexin V and TUNEL assays. (A) Typical scatter dot plot obtained for the samples used in this study. The elliptical highlighted area corresponds with the sperm population, as confirmed using a sorter and analyzing the population by microscopy. (B) Typical plot of a sperm population obtained with the annexin $\mathrm{V}$ assay, combining the fluorescence of annexin $\mathrm{V}$ (An, red
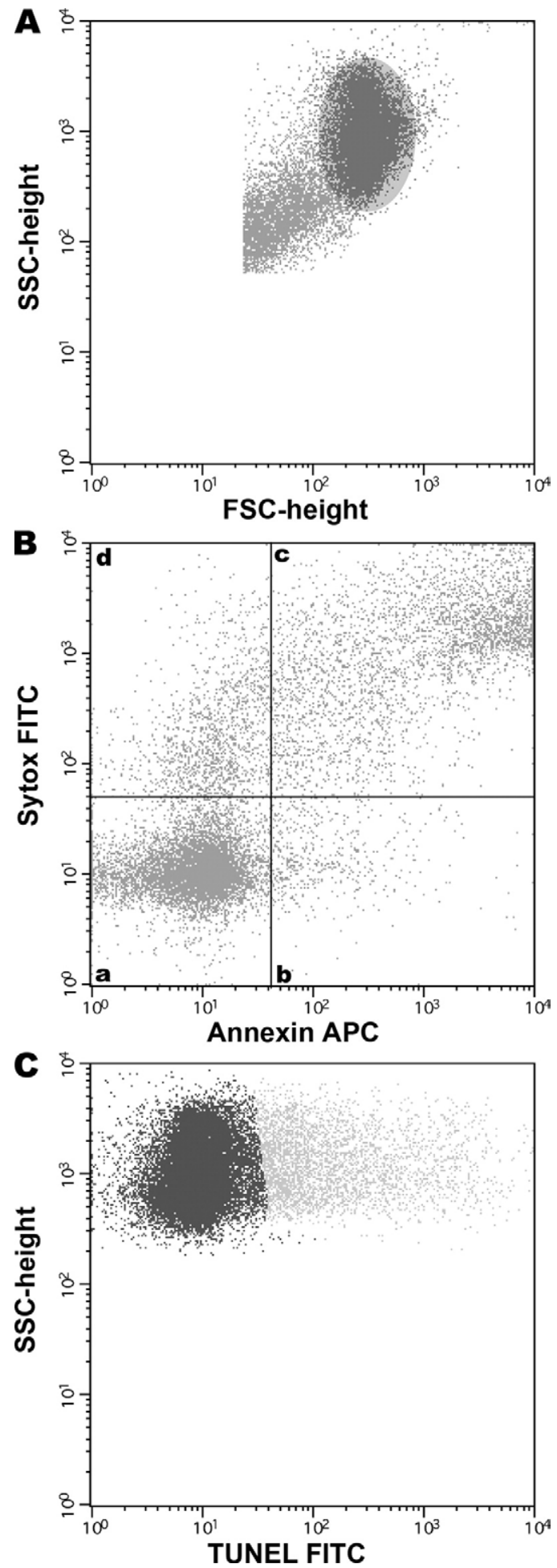

Varum. Assays to monitor human sperm quality. Fertil Steril 2007. 
channel) and Sytox (S, green channel). The quadrants represent (a) viable sperm (An-;S-), (b) sperm in early apoptosis $(\mathrm{An}+; \mathrm{S}-),(c)$ sperm in late apoptosis $(\mathrm{An}+; \mathrm{S}+)$, and $(d)$ nonviable sperm (An-;S+). (C) Typical plot of a sperm population obtained with the TUNEL assay. The dark dots represent unlabeled cells (TUNEL-), the lighter dots represent cells staining in the green channel (TUNEL+). The accuracy regarding the percentages of different cell populations calculated by flow cytometry with both assays was confirmed using fluorescence microscopy on the same samples (see text). FSC, forward scatter (relative size); SSC, side scatter (relative granularity or internal complexity).

\section{Sperm-Ubiquitin Tag Immunoassay}

The sperm-ubiquitin tag immunoassay (SUTI) was essentially carried out as originally defined $(11,13)$. Briefly, the cell suspension $\left(5 \times 10^{6}\right.$ spermatozoa/mL $)$ was fixed with $2 \%$ formaldehyde in PBS for 60 minutes and then centrifuged $(800 \times g$ for 5 minutes). No permeabilization of the sperm membrane was performed. Using the fluorescent lectin Pisum sativum agglutinin (PSA)-FITC, which binds to exposed acrosomal contents, we were able to show that sperm membranes are mostly intact after this treatment (not shown). Samples were blocked in PBS with $2 \mathrm{mg} / \mathrm{mL}$ bovine serum albumin (BSA) and $100 \mathrm{mM}$ glycine (blocking solution). The same procedure was also carried out with sperm previously attached to coverslips for fluorescence microscopy analysis.

After fixation, samples were incubated with anti-ubiquitin antibody (clone KM 691 mouse IgM; Kamiya Biomedical, Seattle, WA), diluted 1:400 in blocking solution, at $37^{\circ} \mathrm{C}$ for 30 minutes; centrifuged $(800 \times g$ for 5 minutes); washed in PBS with $0.1 \%$ Triton for 30 minutes, and centrifuged again. They were then incubated with the appropriate secondary antibody (anti-IgM 488, Molecular Probes) diluted 1:200 $\left(37^{\circ} \mathrm{C}, 20\right.$ minutes). Finally, samples were centrifuged, washed, and resuspended in $500 \mu \mathrm{L}$ of blocking solution. Negative controls were prepared for each experimental sample by omitting the primary antibody.

Samples were analyzed on the day of processing using a FACSCalibur flow cytometer and a fluorescence microscope. SUTI mean values and percentages and histograms of relative fluorescence were obtained using the FACSCalibur cytometer at 488-nm wavelength. In each sample, 10,000 cells were analyzed and the sperm population, ubiquitination means, and percentages were analyzed using CELL Quest and Paint-AGate software. A similar protocol was performed in order to analyse the data by fluorescence microscopy. In this case, SUTI-treated coverslips were placed on slides, and DAPI counterstaining was included to determine the percentage of ubiquitin-positive cells in each sample. For each sample, we assessed at least 200 sperm cells per slide in at least four different fields. Both interobserver and intraobserver variability were $<5 \%$. It is not known whether the anti-ubiquitin antibody, which was raised against ubiquitin monomers, has distinct specificities for mono- or polyubiquitination.

\section{Ubiquitin-Annexin V Assay}

For dual ubiquitin-annexin $\mathrm{V}$ staining, we first carried out the annexin $\mathrm{V}$ protocol, followed by formaldehyde fixation and immunostaining for ubiquitin, including the DAPI counterstain. All procedures were as described above. All samples were analyzed immediately with a fluorescence microscope. We analyzed 200 sperm cells per coverslip to determine percentages of positive cells for surface ubiquitination, positive cells for annexin $\mathrm{V}$, and positive cells for both. The interobserver and intraobserver variability were $<5 \%$.

\section{Merocyanine $\mathbf{5 4 0}$ Staining}

Live sperm in suspension $\left(5 \times 10^{6}\right.$ spermatozoa $\left./ \mathrm{mL}\right)$ were incubated at $37^{\circ} \mathrm{C}$ for 15 minutes at room temperature in HEPES buffer containing $27 \mu \mathrm{M}$ Merocyanine 540 (Molecular Probes). After incubation, the sample was washed with PBS $0.1 \%$ Triton X-100 and examined by fluorescence microscopy. Due to quick photobleaching of the dye, it seemed often useful to omit the washing step and fix the cells. In this case, samples were fixed in $2 \%$ formaldehyde, processed for ubiquitin immunostaining, and observed under fluorescence microscopy, as described above, to evaluate the presence of non-DNA-containing ubiquitin bodies.

It should be noted that, in the current study, Merocyanine 540 was not used to assess sperm function, namely as a possible reporter for changes related to sperm capacitation (15), but only to detect the presence of non-DNA-containing semen bodies, as discussed in previous studies (14),

\section{Statistical Analysis}

The criterion of data normality was evaluated. As all the variables had a normal distribution, parametric tests were performed. Comparisons between classes of patients were carried out by one-way ANOVA for apoptotic levels, DNA fragmentation, and ubiquitination, both for percentages of labeled cells (fluorescence microscopy) and fluorescence means (flow cytometry). Statistical relevance was considered when $P<.05$. We also calculated Pearson's correlation coefficients $(r)(P<.05)$ to determine the correlation between apoptotic levels, DNA fragmentation, ubiquitination fluorescence means (flow cytometry), percentages of ubiquitinlabeled cells (fluorescence microscopy), and seminal param- 


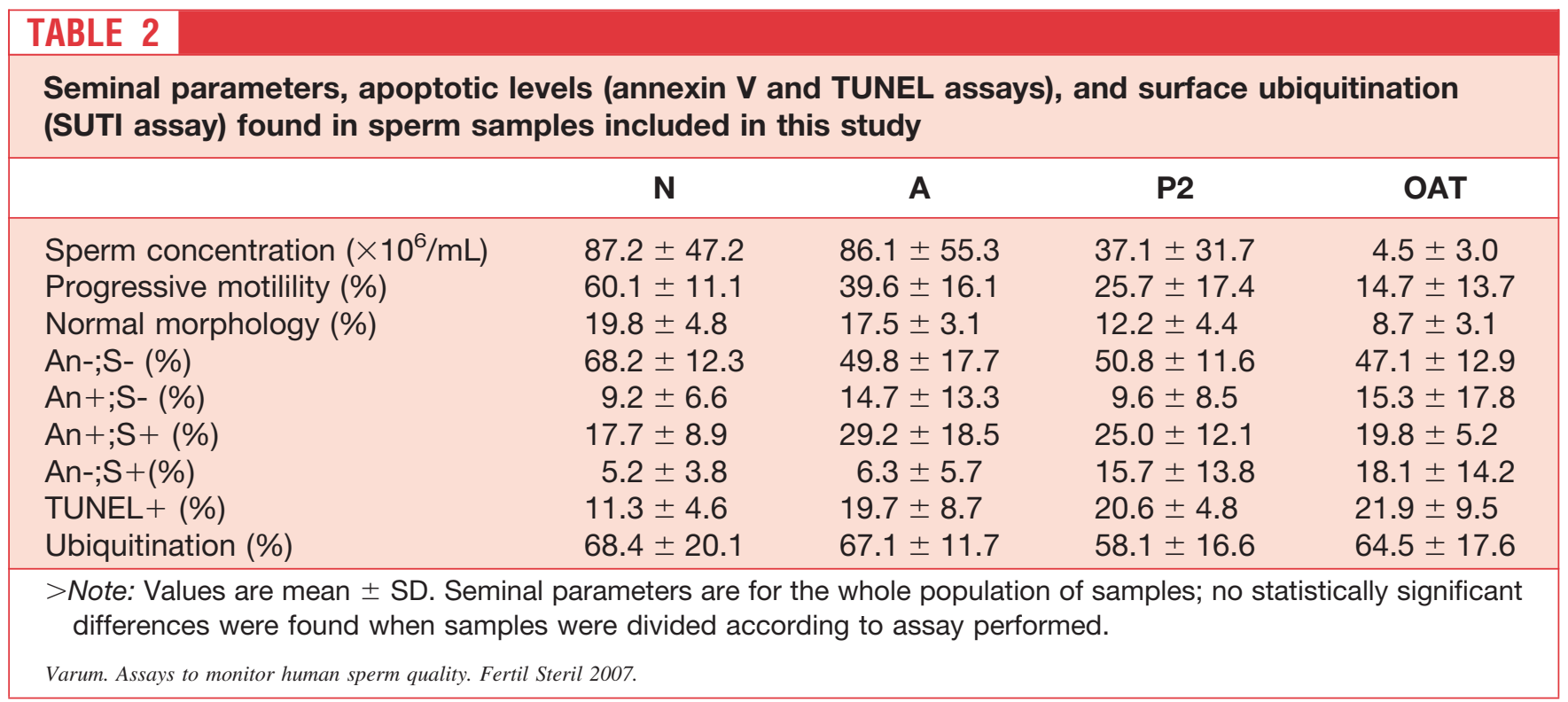

eters. All tests were carried out using Prism 4.0 (Graph Pad Software, San Diego, CA).

\section{RESULTS}

\section{Apoptotic Markers and Semen Quality}

Samples were divided into four groups: normozoospermic $(\mathrm{N})$, asthenozoospermic (A), oligoasthenoteratozoospermic (OAT), and samples that presented two parameters with values lower than those indicated by the World Health Organization (P2). These populations formed statistically separate entities for semen parameters $(P<.0001)$, and differences between them were as expected (e.g., $\mathrm{N}$ and A differed only in motility), except for samples with two abnormal seminal parameters, where subpopulations (i.e., oligoteratozoospermic, asthenoteratozoospermic, oligoasthenozoospermic) could not be distinguished statistically, thus prompting the creation of the $\mathrm{P} 2$ population. The means and standard deviation (SD) for the seminal parameters of samples used are presented in Table 2 .

To assess PS exposure on the outer leaflet of the plasma membrane (plus cell integrity) and DNA fragmentation, we used the annexin $\mathrm{V}$ and the TUNEL assays, respectively. Both assays were analyzed by flow cytometry and fluorescence microscopy.

Figure 1A represents a typical light-scatter flow cytometry diagram, and the tightly focused cluster highlighted represents the sperm population typical of our samples. The population was defined after passing samples through a sorter and analyzing the multiple populations obtained. The lower left and the upper right corners represent small cells/ cellular debris and large cells, respectively.

Figure 1B shows the typical flow cytometry dot plot for annexin $\mathrm{V}$ and Sytox staining found in all samples in which four distinctive cell populations can be seen: [a] viable cells,

\section{FIGURE 2}

Populations of sperm defined by the annexin $\mathrm{V}$ and TUNEL assays observed by fluorescence microscopy. Typical sperm cells detected by the annexin V (A-C) and TUNEL (D) assays (see Materials and Methods). (A-C) Annexin $\mathrm{V}$ is in red and Sytox is in green. Annexin V staining was strong in the head and midpiece (plus cytoplasmic droplets) but also in the tail. (A) Sperm in early apoptosis (An+;S-); (B) sperm in late apoptosis $($ An $+; \mathrm{S}+)$; (C) nonviable sperm (An-;S+). (D) TUNEL+ cells: TUNEL staining is in green and DNA is in blue. Viable sperm cells were not stained with annexin V, Sytox, or TUNEL. Bar = $10 \mu \mathrm{m}$.
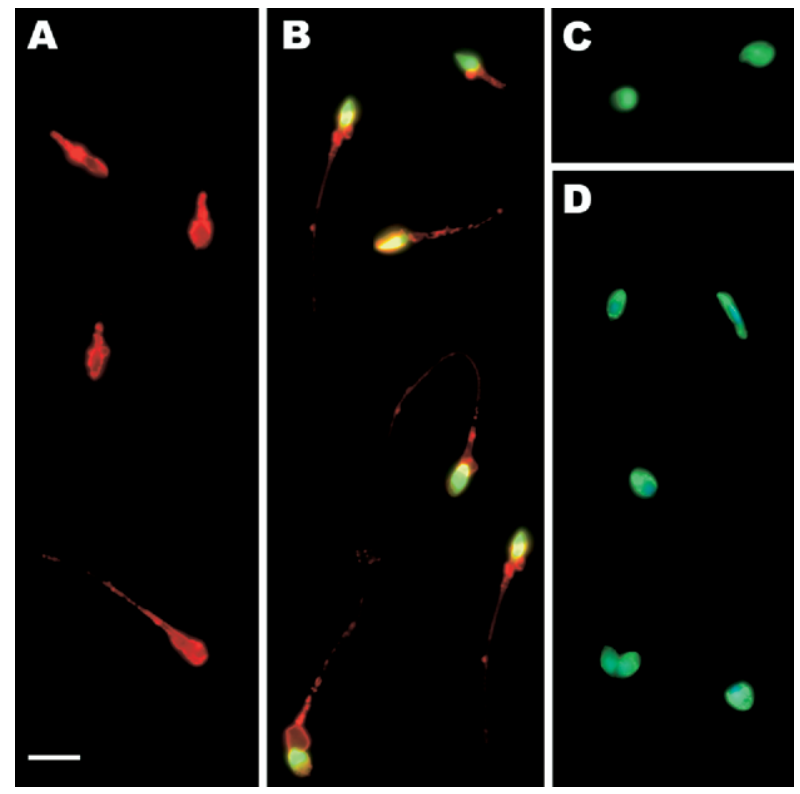

Varum. Assays to monitor human sperm quality. Fertil Steril 2007. 
The annexin $\mathrm{V}$ assay carried out on samples from men with varying etiologies. Normozoospermic $(\mathrm{N})$, asthenozoospermic (A), oligoasthenoteratozoospermic (OAT), and samples with two abnormal seminal parameters (P2) were analyzed by flow cytometry using the annexin $\mathrm{V}$ assay, and the percentages of the four sperm populations were defined by the assay (see Fig. 1). (A) Viable sperm (An-;S-); (B) sperm in early apoptosis $(\mathrm{An}+; \mathrm{S}-)$; (C) sperm in late apoptosis $(\mathrm{An}+; \mathrm{S}+)$; and (D) nonviable sperm (An-;S+). Signs indicate differences relative to $N$ samples in each case $\left({ }^{\star} P<.05 ;{ }^{* \star} P<.01 ;{ }^{* \star \star} P<.001\right)$. See text for further statistically significant comparisons.
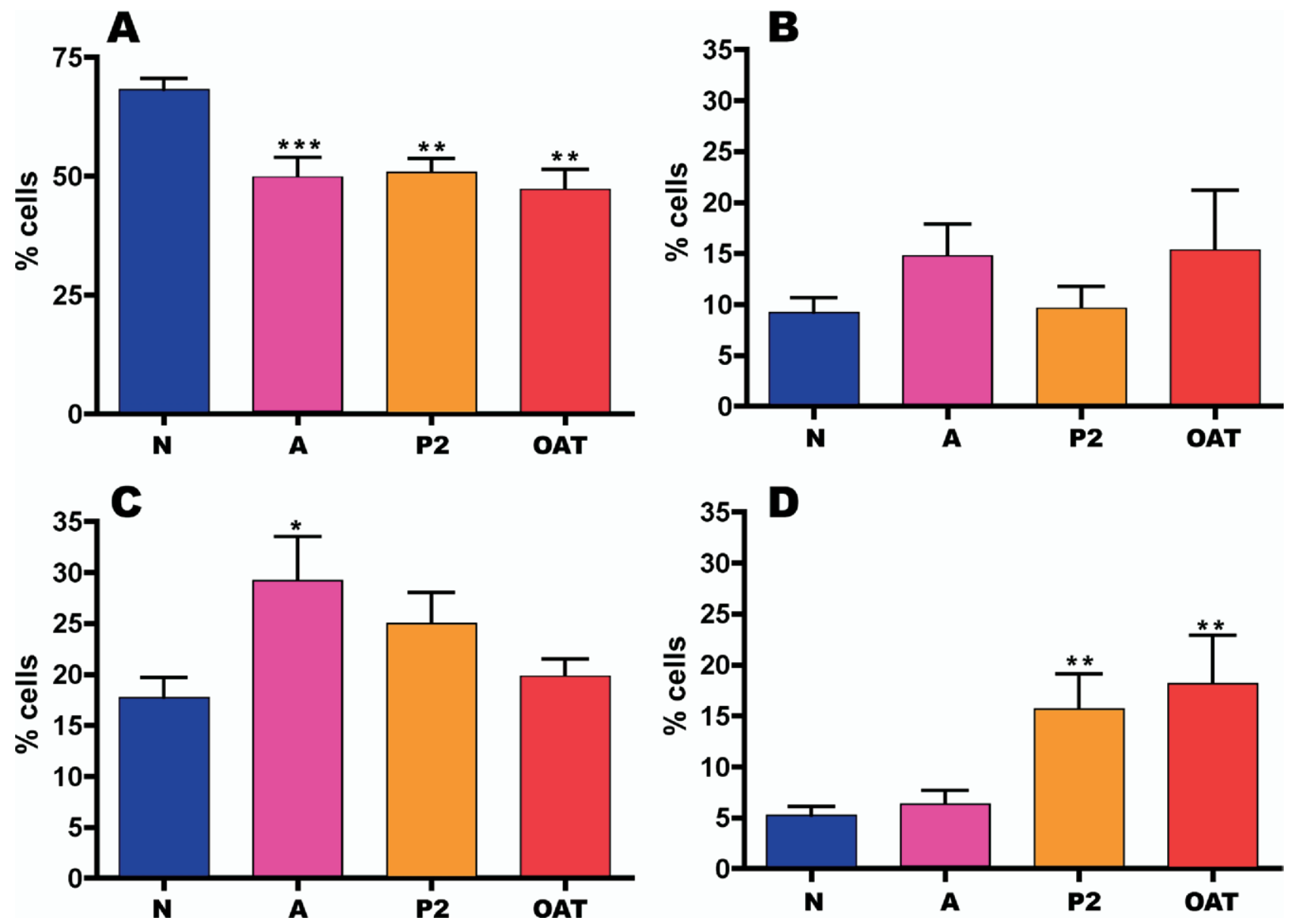

Varum. Assays to monitor human sperm quality. Fertil Steril 2007.

with negative staining for both annexin $\mathrm{V}$ and Sytox (no fluorescence, An-;S-); [b] early apoptotic cells, positive for annexin V but negative for Sytox (red, $\mathrm{An}+; \mathrm{S}-$ ); [c] late apoptotic cells, positive for both annexin $\mathrm{V}$ and Sytox (red and green, $\mathrm{An}+; \mathrm{S}+) ;[\mathrm{d}]$ nonviable cells, positive only for Sytox (green, An-;S+). In Figure 1C, the typical cytometry dot plot for the TUNEL assay found in this study is presented, and it is possible to distinguish two different populations: [i] the left one (dark spots) corresponds with sperm with no DNA fragmentation (no green fluorescence, TUNEL-), [ii] and the one on the right (lighter spots) represents spermatozoa with DNA fragmentation (green fluorescence, TUNEL + ).
To ensure that the percentages of cells calculated by flow cytometry accurately characterized each sample, a simultaneous evaluation using fluorescence microscopy was carried out on the same populations (Fig. 2). The results of these distinct quantifications for both assays were statistically indistinguishable (data not shown).

The annexin V assay allowed the quantification of percentages of viable cells and cells with distinct degrees of apoptosis in the different groups (Table 2; Fig. 3). The means of viable cell populations were significantly different $(P<.0001)$ (Fig. 3A), and Tukey's multiple comparison test indicated that significant differences occur between N/A 
$(P<.001), \mathrm{N} / \mathrm{P} 2 \quad(P<.01)$, and N/OAT $(P<.01)$, but not among A, P2, and OAT. No differences were found among groups relatively to early apoptosis (Fig. 3B; $P>.05$ ). On the other hand, when late apoptosis was considered, the only significant difference found was between $\mathrm{N}$ and $\mathrm{A}$ samples (Fig. 3C; $P<.05$ ). Interestingly, $\mathrm{N}$ and $\mathrm{A}$ samples (as well as OAT and P2) could not be distinguished in terms of nonviable sperm (Fig. 3D). However, $\mathrm{N}$ and A samples differed significantly from both OAT and $\mathrm{P} 2$ populations $(P<.01$ and $P<.05$, respectively).

When comparing annexin $\mathrm{V}$ populations using two-tailed Pearson's correlation test, we found that the percentage of viable cells negatively correlates with early apoptosis $(P=.0104 ; \mathrm{r}=-0.3206)$, with nonviable cells $(P=.0405 ; r$ $=-0.2588)$, and, most strongly, with late apoptosis $(P<.0001 ; r=-0.7259)$. The only other significant correlation was between early apoptosis and nonviable cells $(P=.0008 ; r=-0.4107)$. Thus, early apoptosis seemed to inversely vary with lack of cell viability but was not correlated with late apoptosis $(P=.2817)$, which, in turn, did not correlate with nonviable cells $(P=.804)$.

Percentages of DNA fragmentation between the different groups were calculated using the TUNEL assay coupled to flow cytometry (Table 2). In this case, the only statistically significant differences found were between $\mathrm{N}$ and all the other groups (N/A $P<.01 ;$ N/P2 $P<.001$; N/OAT $P<.01$ ).

To assess the possible impact of sperm apoptosis and DNA fragmentation on sperm quality, we further analyzed the correlations between these indexes and seminal parameters. When comparing results from the annexin $\mathrm{V}$ and TUNEL assays carried out in the same samples, we observed that the only significant (and positive) correlation $(P=.0152$ and $r=0.4192$ ) was found between DNA fragmentation $(\mathrm{TUNEL}+)$ and lack of cell viability $(\mathrm{An}-; \mathrm{S}+)$. The percentage of TUNEL + spermatozoa and of nonviable spermatozoa $(\mathrm{An}-; \mathrm{S}+)$ were negatively and significantly correlated with sperm concentration $(P=.0180$ and $r=-0.3096$; $P=.0258$ and $r=-0.2765$, respectively); percentage of progressively motile cells $(P<.0001$ and $r=-0.5190$; $P=.0301$ and $r=-0.2713$, respectively), and percentage of morphologically normal cells $(P=.0020$ and $r=-0.3973$; $P=.0034$ and $r=-0.3634$, respectively). In contrast, the percentage of viable cells $(\mathrm{An}-; \mathrm{S}-)$ was positively and significantly correlated with both progressive motility $(P=.0097$ and $r=0.3211)$ and normal morphology $(P=.0080$ and $r=0.3313)$.

\section{Surface Ubiquitination, Semen Parameters, and Apoptosis}

The SUTI assay was carried out by flow cytometry and fluorescence microscopy. In flow cytometry experiments, we analyzed both the percentage of ubiquitinated entities and the intensity of fluorescence. In the former case, no differences were found between populations (see Fig. 4B), but in the latter case, samples with at least one abnormal WHO parameter (concentration, motility, morphology) yielded a stronger signal when compared with normal samples, as shown by the respective fluorescence means (Fig. 4A; $P=.0002$ ). This is in agreement with what has been recently reported (11), although in our case we used direct fluorescence means and did not find any need to divide our histograms into quartiles, which could have added another layer of data constriction. On the other hand, similar to what was described for the TUNEL assay, no differences were detected between the different populations with abnormal parameters (A, P2, OAT, not shown).

When correlating ubiquitin fluorescence means with individual sperm parameters, significant inverse correlations were found with morphology $(P=.0028 ; r=-0.4962)$, progressive motility $(P=.0094 ; r=-0.4390)$, and sperm concentration $(P=.0472 ; r=-0.3427)$. Nevertheless, when percentage of ubiquitinated entities was taken into account, no significant correlations between surface ubiquitination and sperm parameters were encountered for concentration $(P=.2935$ and $r=0.1391$; Fig. 4C), progressive motility $(P=.5693$ and $r=0.07559$; Fig. 4D), and normal morphology $(P=.5529$ and $r=0.07882$; Fig. 4E), and $P$ values were always quite high.

This contradicts recent findings (14) and may suggest a lack of reproducibility for this relatively new assay. In order to confirm these results, we used the SUTI assay coupled to fluorescence microscopy (Fig. 4B and F). We found several patterns of labeling, with special incidence on the tail, and determined that both normal and abnormal spermatozoa may or may not be ubiquitinated in any given sample (Fig. 4F). When surface ubiquitination was quantified by percentage of labeled sperm cells, no differences were found between populations $(P=.2130)$, confirming our flow cytometry results (Table 2; Fig. 4B).

Although our flow cytometry and fluorescence microscopy results were in agreement in terms of percentage of ubiquitinated entities, and we know that in the former technique only DNA-containing sperm cells are counted by the observer, we decided to test our samples for the presence of M540-positive bodies. We found that these structures were indeed abundant in OAT samples as described (14) (Fig. $4 \mathrm{H}$ ), but not in all cases where a high ubiquitin mean fluorescence was detected by flow cytometry (e.g., in A samples). Furthermore, in 37 samples tested for M540 (14 N; $8 \mathrm{~A} ; 11 \mathrm{P} 2$; 4 OAT), only in two of the OATs did we find non-DNA-containing structures that were significantly ubiquitinated and that were similar to what has been reported (Fig. 4H and I).

To investigate further the nature of surface ubiquitination, we decided to monitor ubiquitin staining together with the annexin $\mathrm{V}$ assay to determine if ubiquination was related to PS exposure (annexin V). Both assays were tested in the same samples to make sure that the percentage of ubiquitinated cells and annexin V-positive cells did not change when the techniques were carried out separately or simulta- 


\section{FIGURE 4}

Sperm ubiquitination (SUTI assay) monitored by flow cytometry and fluorescence microscopy on samples from men with varying etiologies: correlations with semen parameters, annexin $V$ staining, and Merocyanine 540-positive bodies. (A) Fluorencence means determined by flow cytometry. A clear statistically significant difference was found between normozoospermic $(\mathrm{N})$ samples and all other samples, with at least one abnormal semen parameter $(\# P=.0002)$, but no differences were found between asthenozoospermic $(A)$
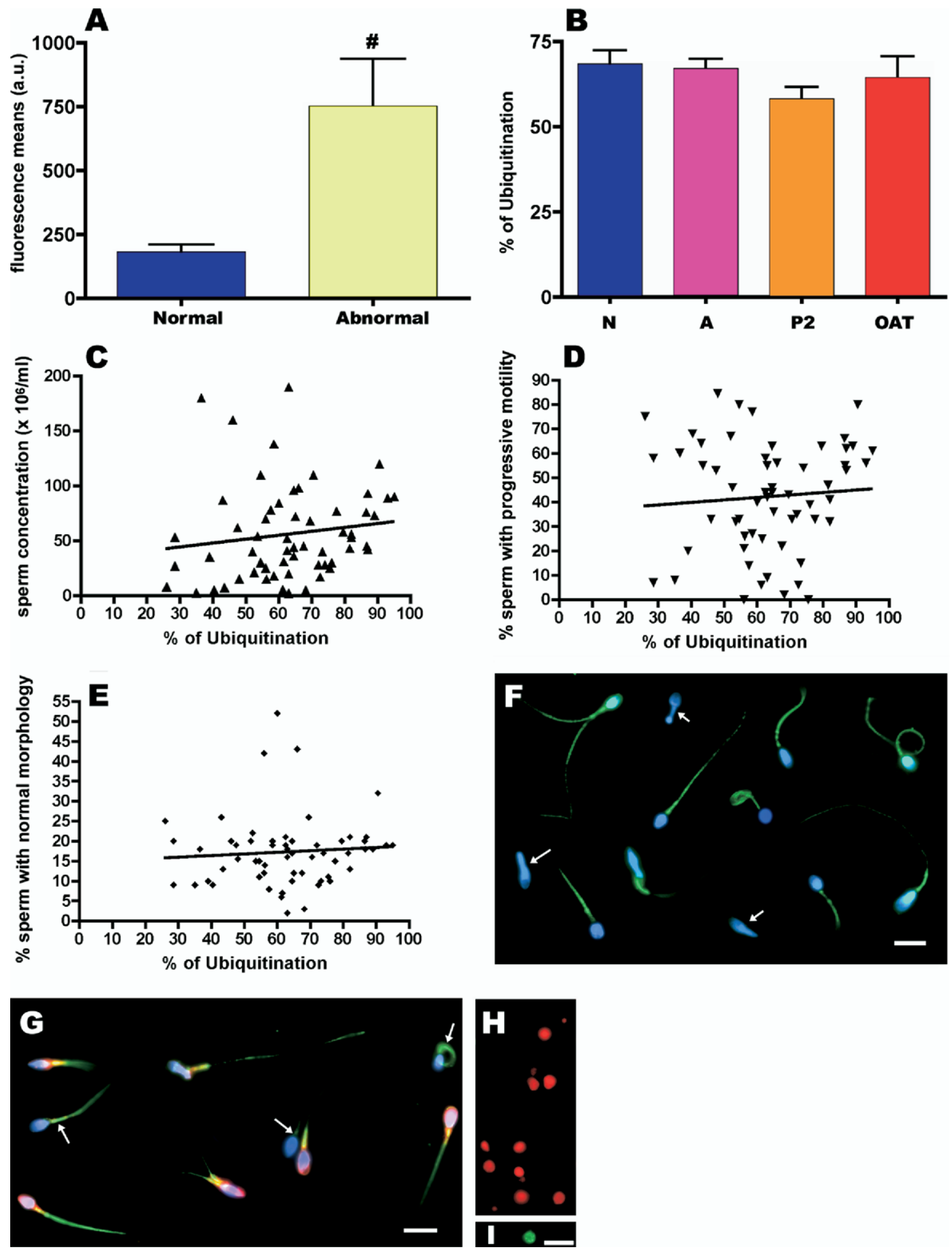

Varum. Assays to monitor human sperm quality. Fertil Steril 2007. 
samples, samples with two abnormal semen parameters (P2), or oligoasthenoteratozoospermic (OAT) samples. (B) Percentages of SUTI-labeled sperm cells in the four populations of samples tested (N; A; P2; OAT). No differences were found between populations $(P=.213)$. (C-E) Correlations between the percentage of ubiquitinated sperm cells and sperm concentration (C; $P=.2935$ and $r=0.1391$ ), progressive motility (D; $P=.5693$ and $r=0.07559$ ), and normal morphology (E; $P=.5529$ and $r=0.07882$ ) were found not to be significant using the two-tailed Pearson's correlation test. (F) Typical examples of sperm labeled with the SUTI assay. Both normal and abnormal cells stain positive for ubiquitin (green), although some obviously abnormal cells are negative (arrows). (G) Sperm cells stained with both annexin V (red) and ubiquitin (green; see Materials and Methods). Annexin V-positive cells were always ubiquitinated, but the reverse was not true (arrows). (H) OAT sample stained with Merocyanine 540 (M540; red) showing many M540-positive bodies without DNA. (I) OAT sample with an ubiquitinated entity (green) that did not contain DNA. This type of structure was found in two of four OATs and not in other samples. (F-I) DNA is in blue. Bars $=10 \mu \mathrm{m}$.

neously (not shown). Although some obviously defective cells were not ubiquitinated (Fig. 4F), all annexin V-positive sperm cells were indeed ubiquitinated under fluorescence microscopy (Fig. 4G). However, we found no statistically significant correlations between surface ubiquitination and any of the four cell populations defined by the annexin $\mathrm{V}$ assay or between ubiquitin staining and TUNEL + cells (Table 3; $P$ values from 0.1635 to 0.7477 ). In all cases, the three assays were performed on the same samples.

\section{DISCUSSION}

Our results show that seminal parameters are inversely and significantly correlated with DNA damage (TUNEL + ) and percentage of nonviable cells (An-;S+). This is in accordance with other authors $(4,18)$, who reported the same correlations between DNA damage and semen parameters.

On the other hand, the percentage of viable cells (An-;S-) correlates positively and significantly with motility and morphology. Furthermore, in our hands TUNEL + is positively and significantly correlated with nonviable cells but not with apoptosis, contrary to other reports showing correlations between TUNEL + cells and late apoptosis (7). However, if we consider that lack of cellular viability follows apoptosis, our results may be in agreement, given the suggestion that
DNA fragmentation only occurs in later stages of sperm apoptosis (7). The absence of correlations between DNA fragmentation and apoptosis (annexin V-positive cells) was also reported by others, and it may occur because the presence of PS in the outer leaflet and DNA fragmentation are events that take place at distinct stages (9). On the other hand, contrary to other authors (9), who reported an inverse correlation between annexin $\mathrm{V}$-positive cells and sperm concentration and motility, our results show no correlation between these cells and seminal parameters.

When samples were divided into groups according to semen parameter criteria, results were in accordance with what has been described. $\mathrm{N}$ men presented a higher and statistically different percentage of viable spermatozoa compared with A, P2, and OAT men. Interestingly, there was a higher percentage of nonviable spermatozoa in OAT and P2 groups than in $\mathrm{N}$ and $\mathrm{A}$, which translates to a higher correlation between lack of viability and morphology. The fact that in A samples the prevalence is for late apoptosis, while OAT and P2 samples have a higher number of nonviable cells, suggests that spermatozoa of the latter samples initiate apoptosis earlier, or that it occurs more rapidly in these groups, considering that early apoptosis, late apoptosis, and nonviability are likely to be sequential events. As expected,

\section{TABLE 3}

Lack of correlation between surface ubiquitination and populations defined by the annexin $\mathbf{V}$ and TUNEL assays

\begin{tabular}{|lccccc|}
\hline & $\begin{array}{c}\text { Viable } \\
\text { (An-;S-) }\end{array}$ & $\begin{array}{c}\text { Early apoptosis } \\
\text { (An+;S-) }\end{array}$ & $\begin{array}{c}\text { Late apoptosis } \\
\text { (An+;S+) }\end{array}$ & $\begin{array}{c}\text { Necrosis } \\
\text { (An-;S+) }\end{array}$ & TUNEL+ \\
\hline$P$ value & .2834 & .1635 & .3472 & .7477 & .169 \\
$r$ value & 0.3220 & -0.4105 & -0.2839 & 0.09898 & -0.3095 \\
\hline
\end{tabular}

$>$ Note: The assays were carried out independently on the same samples using fluorescence microscopy $(n=13$ for ubiquitin/annexin $\mathrm{V}, \mathrm{n}=22$ for ubiquitin/TUNEL). Values for $P$ and $\mathrm{r}$ were calculated using the two-tailed Pearson's correlation test. See Materials and Methods for experimentalal details.

Varum. Assays to monitor human sperm quality. Fertil Steril 2007. 
we also found statistically significant inverse correlations between DNA fragmentation and semen parameters (19).

Results with the SUTI assay were less clear because different conclusions can be reached, depending on whether the percentage of ubiquitinated cells or the means of ubiquitin fluorescence are considered. Using SUTI fluorescence means determined by flow cytometry, we could clearly separate $\mathrm{N}$ samples from all the other groups (but not distinguish between samples with different abnormal sperm parameters), which is in accordance with what has been recently described $(11,14)$. However, when percentages of ubiquitinated entities were considered, both by flow cytometry and fluorescence microscopy, no statistically significant difference between groups was found. Furthermore, no correlation between percentage of surface ubiquitination and seminal parameters was evident, which is not in agreement with the recently reported positive correlation between ubiquitination and semen parameters (especially normal morphology) and the suggestion that surface ubiquitination may actually have a positive influence in sperm function (14).

Two possible explanations can be considered in order to explain the discrepancy regarding correlations between sperm parameters and mean ubiquitin fluorescence (flow cytometry) and lack of correlation with percentage of ubiquitinated cells (flow cytometry and fluorescence microscopy): [a] the fluorescence of labeled sperm cells is more intense in samples with abnormal WHO parameters and thus is picked up by the flow cytometer in terms of fluorescence means, but it is not translated into differences in percentages of labeled cells; [b] something else in the semen (but not sperm) is being ubiquitinated, thus causing samples with abnormal WHO parameters to show a higher fluorescence in the flow cytometer. Although intensity of staining of individual cells did not seem to be very different between samples, the first hypothesis cannot be discounted. In terms of the second possibility, it was proposed (14) that M540positive semen bodies (Fig. 4H) may account for the high ubiquitination rate monitored with flow cytometry, especially in severe OAT samples. However, in our hands and samples, M540-positive bodies did not seem to explain higher ubiquitin fluorescence means in samples with abnormal WHO parameters, certainly not in all cases.

Furthermore, no significant correlation between ubiquitin and apoptotic markers (annexin $\mathrm{V}$ and TUNEL) was detected, contrary to what was found in other species, such as in bull sperm, where a high correlation between TUNEL + and ubiquitinated spermatozoa was reported (20). Even though this result suggests that ubiquitination and apoptosis are independent processes, all cells labeled with annexin $\mathrm{V}$ were ubiquitinated when dual ubiquitin-annexin $\mathrm{V}$ staining was carried out. Finally, and although it has been proposed that ubiquitin is a universal marker for sperm abnormalities (13), we found many clearly abnormal spermatozoa that were not ubiquitinated (Fig. 4F).
Considering these results, and although differences between patient populations cannot be discounted and may explain some of the reported discrepancies, we suggest that surface sperm ubiquitination is not determined by morphologic abnormalities alone and that whatever its biological significance, there must be other factors that trigger surface ubiquitination.

In summary, viable cells, nonviable cells, DNA packaging, and ubiquitin fluorescence intensity means (but not ubiquitinpositive cell number) correlate with seminal parameters. None of the apoptotic markers correlate with ubiquitin labeling in human sperm. The fact that other factors (not only morphology) seem to determine surface ubiquitination in sperm, as well as the existence of major variations between published results, indicates possible issues in assay reproducibility. In conclusion, biological markers, such as those related to sperm integrity and the state of sperm DNA, can potentially be used to separate sperm populations with distinct fertilizing potentials. This is certainly not yet the case for both annexin V surface binding or surface ubiquitination.

Acknowledgments: S.V., C.B., A.P.S., and C.G.S. contributed equally to this study. We thank Dr. Ana Barbosa, Dr. Raquel Brito, Conceição Dias, Fátima Veríssimo (University Hospitals of Coimbra), Alexandra Amaral, and Joana Gonçalves (Center for Neuroscience and Cell Biology) for many helpful discussions and technical assistance, and Prof. Agostinho Almeida Santos (University Hospitals of Coimbra) for continuous encouragement and support. We also thank Sandra Gamboa, Paula C. Mota, and other members of the lab for many fruitful discussions.

\section{REFERENCES}

1. Ozanon C, Chouteau J, Sutovsky P. Clinical adaptation of the sperm ubuquitin tag immunoassay (SUTI): relationship of sperm ubiquitylation with sperm quality in gradient-purified semen samples from 93 men from a general infertility clinic population. Hum Reprod 2005;20: 2271-8.

2. World Health Organization. WHO Laboratory manual for the examination of human semen and sperm-cervical mucus interaction. 4th ed. Cambridge: Cambridge University Press, 1999.

3. Evenson DP, Larson KJ, Jost LK. Sperm chromatin structure assay: its clinical use for detecting sperm DNA fragmentation in male infertility and comparisons with other techniques. J Androl 2002;23:25-43.

4. Gandini L, Lombardo F, Paoli D, Caponecchia L, Familiari G, Verlengia C, et al. Study of apoptotic DNA fragmentation in human spermatozoa. Hum Reprod 2000;15:830-9.

5. Sakkas D, Mariethoz E, Manicardi G, Bizzaro D, Bianchi P, Bianchi U. Origin of DNA damage in ejaculated human spermatozoa. Rev Reprod 1999;4:31-7.

6. Sinha Hikim AP, Swerdloff RS. Hormonal and genetic control of germ cell apoptosis in the testis. Rev Reprod 1999;4:38-47.

7. Shen HM, Dai J, Chia SE, Lim A, Ong CN. Detection of apoptotic alterations in sperm in subfertile patients and their correlations with sperm quality. Hum Reprod 2002;17:1266-73.

8. Barroso G, Morshedi M, Oehninger S. Analysis of DNA fragmentation, plasma membrane translocation of phosphatidylserine and oxidative stress in human spermatozoa. Hum Reprod 2000;15:1338-44.

9. Oosterhuis GJE, Mulder AB, Kalsbeek-Batenburg E, Lambalk CB, Schoemaker J, Vermes I. Measuring apoptosis in human spermatozoa: a biological assay for semen quality? Fertil Steril 2000;74:245-50.

10. Muratori M, Piomboni P, Baldi E, Filimberti E, Pecchioli P, Moretti E. Functional and ultrastructural features of DNA-fragmented human sperm. J Androl 2000;21:903-12. 
11. Sutovsky P, Hausser R, Sutovsky M. Increased levels of sperm ubiquitin correlate with semen quality in men from an andrology laboratory clinic population. Hum Reprod 2004;19:628-38.

12. Hochstrasser M. Ubiquitin - dependent protein degradation. Annu Rev Genet 1996;30:405-39.

13. Sutovsky P, Terada Y, Schatten G. Ubiquitin-based sperm assay for the diagnosis of male factor infertility. Hum Reprod 2001;16:250-8.

14. Muratori M, Marchiani S, Forti G, Baldi E. Sperm ubiquitination positively correlates to normal morphology in human semen. Hum Reprod 2005;20:1035-43.

15. Muratori M, Porazzi I, Luconi M, Marchiani S, Forti G, Baldi E. Annexin $\mathrm{V}$ binding and merocyanine staining fail to detect human sperm capacitation. J Androl 2004;24:797-810.

16. Mota PC, Ramalho-Santos J. Comparison between different markers for sperm quality in the cat: Diff-Quik as a simple optical technique to assess changes in the DNA of feline epididymal sperm. Theriogenology 2006;65:1360-75.

17. Kruger TF, Menkveld R, Stander FS, Lombard CJ, Van der Merwe JP, Van Zyl JA, et al.. Sperm morphologic features as a prognostic factor in in-vitro fertilization. Fertil Steril 1986;46:1118-23.

18. Irvine DS, Twigg JP, Gordon EL, Fulton N, Milne PA, Aitken RJ. DNA integrity in human spermatozoa: relationships with semen quality. J Androl 2000;21:33-43.

19. Huang CC, Lin DP, Tsao HM, Cheng TC, Liu CH, Lee MS. Sperm DNA fragmentation negatively correlates with velocity and fertilization rates but might no affect pregnancy rates. Fertil Steril 2005; 84:130-40.

20. Sutovsky P, Neuber E, Schatten G. Ubiquitin - dependent sperm quality control mechanism recognizes spermatozoa with DNA defects as revealed by dual ubiquitin-TUNEL assay. Mol Reprod Dev 2002;61:406-13. 